RESEARCH ARTICLE

\title{
Nosocomial Infections in Very Low Birth Weight Infants: Etiology and Risk Factors
}

\author{
V. Vamsi Sivarama Raju, Jayashree Purkayastha, Leslie Edward Lewis, Ramesh Bhat Y. \\ Department of Pediatrics, Kasturba Medical College, Manipal, Manipal University, Karnataka, India
}

\section{ABSTRACT}

Objective: We aimed to find out the incidence and risk factors of nosocomial infections in VLBW neonates and to explore the microbiologic flora, sensitivity pattern and outcome.

Methods: A prospective, observational study was performed in a neonatal intensive care unit of a teaching hospital. VLBW infants excluding those diagnosed with early onset sepsis, admitted to the unit between August 2012 and March 2013 were followed up for evidence of nosocomial sepsis.

Results: Of 92 VLBW infants, 23 developed nosocomial sepsis, incidence rate being $25 \%$. Decreasing birth weight (1251-1500 g, 10.9\%; 1001-1250 g, 28\%; 751-1000 g, 50\%; P<.001) and gestational age (>33wks, 0\%; 29-32wks, $21.3 \% ; 26-28 \mathrm{wks}, 66.7 \% ; \mathrm{p}<.001)$ were found to be associated with statistically significant increase in nosocomial sepsis rate.

On multivariate logistic regression, only peripherally inserted central catheter (PICC) line was independently associated with increased risk of nosocomial sepsis (aOR 13.33, 95\% Cl 3.58-49.5) in VLBW.

A predominance of Gram negative over Gram positive nosocomial sepsis (75\% vs. $25 \%$ ) with higher mortality in the Gram negative group (55\% vs. $0 \%$ ) was observed. Klebsiella pneumoniae was the predominant microbe (33.4\%). All microbes were sensitive to first line antibiotics except Elizabeth kingia meningoseptica and one episode of $K$. pneumoniae. Seven (30.4\%) VLBW neonates with nosocomial sepsis died.

Conclusions: Among VLBW infants, the incidence of nosocomial sepsis was 25\%. Lower birth weight, lesser gestational age and PICC line were important risk factors. Gram negative nosocomial sepsis was associated with higher mortality compared to Gram positive sepsis. J Microbiol Infect Dis 2017; 7(1): 7-12

Keywords: Neonates; Nosocomial sepsis; VLBW infants

\section{INTRODUCTION}

Very low birth weight $(<1500$ g) newborns experience higher morbidity and mortality. Besides their immaturity, morbidity patterns vary due to multiple factors. Infections especially, nosocomial infections among very low birth weight (VLBW) neonates not only influence neonatal survival but also add to cost of therapy $[1,2]$. Nosocomial infections may affect up to $40 \%$ of infants seeking intensive care unit (NICU) admissions [3-5].Immature immune system, exposure to invasive procedures and increased hospital stay predispose VLBW neonates to nosocomial infections $[1,6,7]$.
Sepsis is estimated to account for $30-50 \%$ of total neonatal deaths each year in developing countries [8].The immature humoral and cellular immunity and lower production of immunoglobulins than adults increases their risk to sepsis. Transfer of maternal antibodies across placenta mediate humoral immunity primarily, hence VLBW premature infants are less likely to receive as many immunoglobulins as term infants. In addition, neonates also have less efficient T-cell function [9]. The functions of complement and phagocytic system including phagocytosis, phagocyte migration and toxin production are also deficient in neonates [10]. 
By virtue of the prolonged hospitalization, VLBW infants are at prolonged risk for acquiring infections, particularly nosocomial infections. The strategies to reduce and prevent infections in these infants obviously decrease mortality and morbidity. We aimed to study characteristics of nosocomial infections in VLBW infants along with identification of risk factors for the same. We also aimed to explore the microbiologic flora, their sensitivity pattern and the short term outcome of VLBW infants with nosocomial sepsis.

\section{METHODS}

All VLBW infants who were hospitalized in a teaching hospital between August 2012 to march 2013 and survived for 3 days or more were prospectively followed up for evidence of nosocomial sepsis. This teaching hospital has a level 2 neonatal intensive care unit (NICU) with facilities such as radiant warmers, incubators, vital signs monitors, total parenteral nutrition facility with laminar flow, ventilators (Drager Babylog and SLE 5000), infusion pumps and a team of excellent pediatric surgeons. The unit has about 1500 admissions annually. For nosocomial sepsis, we used the definition given by Centers for Disease Control and Prevention [11]. Neonates with onset of sepsis within three days of life were excluded. All the infants were managed as per the unit protocol. The routine NICU protocol among such infants includes a sepsis screen and blood culture. Cerebrospinal fluid (CSF) analysis and culture was done in neonates who had positive blood culture or based on the clinical decision of the treating physician. Urine culture, and a chest radiograph (CXR) was taken whenever needed. Samples of blood, CSF and urine were processed and analyzed according to standard bacteriological procedures. The neonates were empirically started on a combination of Penicillin and an Aminoglycoside, usually Ampicillin and Amikacin in case of suspected sepsis pending sepsis screen/blood culture reports. If the infant received these antibiotics within 72 hours of the sepsis screen, second-line antibiotics were prescribed. The routine unit practice among infants below 1300 grams was to secure a peripheral line on day one and to start on IV fluids and aminoacids (partial parenteral nutrition, PPN). On day two or day three of life a peripherally inserted central catheter (PICC) line was secured and total parenteral nutrition (TPN) was started. Enteral feeding with breast milk was initiated at the earliest and graded up as per standard protocol. We had not used umbilical lines in these groups of neonates.

We collected the demographic, clinical and laboratory data on all VLBW infants. Neonatal details included place and date of birth, gestational age, mode of delivery, resuscitation methods and Apgar score, gender, birth weight, age at sepsis screening, blood culture reports, physical examination details and antibiotics used. Maternal details included age and parity.

Details about nosocomial sepsis, nosocomial pneumonia, necrotizing enterocolitis (NEC), meningitis if any were collected. Proven risk factors for nosocomial sepsis like peripheral venous catheter, PICC lines, Endotracheal (ET) tube/ Continuous positive airway pressure (CPAP) (number of days of ventilation), Patent Ductus Arteriosus (PDA), Intra Ventricular hemorrhage(IVH), Broncho Pulmonary Dysplasia (BPD) along with duration of hospital stay were collected. For the study, we obtained an approval from the institutional ethics committee.

SPSS software package 16 was used for statistical analysis. The association of risk factors such as ventilation requirement, presence of peripheral and PICC lines and TPN administration with nosocomial sepsis was analyzed using Chi square test. Odds ratio was used to denote the association between probable risk factors and nosocomial infection in VLBW infants. A multivariate logistic regression analysis using forward Wald was used to further explore the relationship of these risk factors with nosocomial sepsis. The relationships between selected morbidities and nosocomial sepsis were also similarly assessed using Chi square test. The relationship of continuous data such as duration of mechanical ventilation, duration of PICC lines, and duration of TPN days and length of NICU stay with nosocomial sepsis were assessed using Mann-Whitney $\mathrm{U}$ test.

\section{RESULTS}

A total of 92 VLBW infants had satisfied the inclusion criteria and 23 infants among them were diagnosed to have nosocomial sepsis with an incidence rate of $25 \%$. Median birth weight of 
study neonates with nosocomial sepsis was 1030 (range:870-1205) grams. Median gestation of study neonates with nosocomial sepsis was 29 (range: 27-31) weeks.

Out of 47 male VLBW neonates enrolled in the study, $12(25.5 \%)$ had nosocomial sepsis and out of 45 female neonates, 11 (24.4\%) had nosocomial sepsis. Nosocomial sepsis in male and female neonates was found to be similar. Rate of nosocomial sepsis among intramural and extramural VLBW neonates was similar with $24.6 \%$ (17 out of 69 ) and $26.08 \%$ (6 out of 23 ) respectively.

The incidence of nosocomial sepsis in neonates with birth weight of $751-1000 \mathrm{~g}$ was $50 \%$; with birth weight of $1001-1250 \mathrm{~g}$ was $28 \%$ and with birth weight of $1251-1500 \mathrm{~g}$ was $10.9 \%$. This difference was statistically significant $(P<.001)$. The incidences of nosocomial sepsis in neonates with gestational age of 26-28 wks., 2932 wks. and $33+$ wks. were $66.7 \%, 21.3 \%$ and $0 \%$ respectively. This difference was statistically significant $(p<.001)$
In the univariate analysis, invasive mechanical ventilation (OR $4.59,95 \% \mathrm{Cl} 1.68-12.52$ ), PICC line (OR 13.33, 95\%Cl 3.58-49.54), TPN (OR $11.73,95 \% \mathrm{Cl} 3.16-43.44)$ and presence of PDA (OR $7.76,95 \% \mathrm{Cl} 1.75-34.27$ ) were associated with significantly higher risk for nosocomial sepsis(Table 1 and 2). However, in the multivariate logistic regression analysis using forward Wald, only PICC line was found to be independently associated with increased risk of nosocomial sepsis in VLBW infants (aOR 13.33, 95\% Cl 3.58-49.5).

On comparison of total duration of invasive mechanical ventilation, non-invasive ventilation $(\mathrm{CPAP}+\mathrm{n}$ SIMV), PICC line, TPN, NICU stay and total hospital stay between infants with and without nosocomial sepsis, we found that the total duration of non-invasive ventilation was significantly higher in infants with nosocomial sepsis as compared to infants without nosocomial sepsis (median of $240 \mathrm{~h}$ and $72 \mathrm{~h}$ respectively, $p=.014$ ) (Table 3 )

Table 1. Baseline characteristics of the study population.

\begin{tabular}{l|cccc}
\hline Interventions & $\begin{array}{c}\text { Sepsis, } \mathbf{n = 2 3 ,} \\
(\%)\end{array}$ & $\begin{array}{c}\text { No sepsis, } \mathbf{n = 6 9 ,} \\
(\%)\end{array}$ & OR (95\% Cl) & $\begin{array}{c}\mathbf{p} \\
\text { value }\end{array}$ \\
\hline Invasive mechanical ventilation $(\mathrm{n}=\mathbf{3 5})$ & $15(65.2)$ & $20(28.9)$ & $4.59(1.7-12.5)$ & 0.003 \\
\hline Non-invasive ventilation $(\mathrm{n}=60)$ & $19(82.6)$ & $41(59.4)$ & $3.24(0.99-10.56)$ & 0.051 \\
Peripheral line $(\mathrm{n}=91)$ & $23(100)$ & $68(98.5)$ & $(0(0.0)$ & 1.00 \\
PICC line $(\mathrm{n}=43)$ & $20(86.9)$ & $23(33.3)$ & $\begin{array}{c}13.33(3.58- \\
49.54)\end{array}$ & $<0.001$ \\
\hline
\end{tabular}

Table 2. Relationship between selected morbidities and nosocomial sepsis.

\begin{tabular}{lcccc}
\hline Risk factor & Sepsis $\mathbf{n = 2 3}(\%)$ & No sepsis $\mathbf{n = 6 9}(\%)$ & OR (95\%Cl) & p value \\
\hline RDS $(n=59)$ & $17(73.9)$ & $42(60.8)$ & $1.82(0.63-5.19)$ & 0.262 \\
$\operatorname{PDA}(n=9)$ & $6(26.1)$ & $3(4.3)$ & $7.76(1.75-34.27)$ & 0.007 \\
$\operatorname{IVH}(n=5)$ & $2(8.6)$ & $3(4.3)$ & $2.09(0.32-13.39)$ & 0.435 \\
$\operatorname{NEC}(n=1)$ & $1(4.3)$ & $0(0)$ & NA & 1.000 \\
BPD $(n=1)$ & $0(0)$ & $1(1.4)$ & NA & 1.000 \\
\hline
\end{tabular}

Table 3. Relationship between duration of care interventions and length of stay with nosocomial sepsis rate..

\begin{tabular}{lccc}
\hline Interventions & Sepsis, Median (IQR) & No sepsis, Median (IQR) & P value \\
\hline Invasive mechanical ventilation (in hours) & $48(24-96)$ & $48(16.50-114)$ & 0.592 \\
Non-invasive ventilation (in hours) & $240(105-432)$ & $72(48-216)$ & 0.014 \\
PICC line (in days) & $15.50(5.25-20)$ & $10(8-12)$ & 0.261 \\
TPN (in days) & $10(4.25-13.75)$ & $7(4-10)$ & 0.371 \\
Duration of NICU stay (in days) & $28(17-47)$ & $23(18-34)$ & 0.138 \\
\hline
\end{tabular}


The nosocomial sepsis during study period were predominantly (75\%) caused by Gram-negative bacteria (Table 4). Klebsiella pneumoniae was the leading causal microbe constituting $33.4 \%$ of all culture positive cases. Gram positive organisms constituted $25 \%$ of all culture positive nosocomial sepsis. Mortality rate among VLBW infants with nosocomial Gram negative sepsis was high (55\%) compared to Gram positive sepsis $(0 \%)$. All the isolated microbes were sensitive to first line antibiotics except Elizabeth kingia meningoseptica and one episode of Klebsiella pneumoniae which were multidrug resistant (MDR) strains (Table 4). Overall 7 $(30.4 \%)$ out of 23 VLBW neonates with nosocomial sepsis died, among these 5 had positive blood cultures, all being Gram negative sepsis.

\section{DISCUSSION}

Nosocomial / late onset sepsis continues to be an important and life threatening complication among VLBW infants [12,13]. In the present study, we prospectively enrolled VLBW neonates to study the incidence and risk factors of nosocomial infections, pattern and sensitivity of microbiological flora and their immediate outcome. Late onset sepsis (LOS) in VLBW neonates occurs in high percentages and the reported rate is $16 \%$ [13] to $25 \%$ [14] in the United States and $30 \%$ in Israel [15]. The incidence rate of nosocomial sepsis among VLBW neonates in our study was $25 \%$. We found that the rate of nosocomial sepsis increased when the birth weight and gestational age decreased, similar to studies by Barbara $\mathrm{J}$ et al [16] and Makhoul et al [15].

Risk factors like mechanical ventilation, umbilical arterial line (UAC), umbilical venous line (UVC), PICC line and TPN were reported to be significant independent predictors of LOS in a study done by Barbera et al [16]. Makhoul et al [15] have reported a significant association of PDA, NEC and BPD with nosocomial infections among VLBW neonates.

In the present study, among various interventions analyzed using univariate analysis, invasive mechanical ventilation (OR $4.59 ; \mathrm{Cl}$ 1.68-12.52), PICC line (OR 13.33; CI 3.5849.54) \& TPN (OR 11.73; Cl 3.16-43.44) were found to be significantly associated with nosocomial sepsis in VLBW neonates. We also found that VLBW infants having PDA were significantly more prone (OR $7.76 ; 1.75-34.27$ ) for nosocomial sepsis. However, on multiple logistic regressions only PICC line (aOR 13.33; $\mathrm{Cl}$ 3.58-49.5) emerged as an independent risk factor for of nosocomial sepsis in VLBW neonates. The difference in risk factors compared to other studies can be explained partly due to minor variations in treatment strategies. Secondly, the sample size of this study is small as it is a single center study over 8 months unlike the earlier reported multicenter studies across two different countries.

The distribution of pathogens in our study was different from those of Barbara $J$ et al [16] and Makhoul et al [15] who reported Gram positive organisms of $70.2 \%$ and $55.4 \%$, Gram negative organisms of $17.6 \%$ and $31.2 \%$, and fungal organisms of $12.2 \%$ and $11.1 \%$ respectively. Overall we isolated a higher percentage of Gram negative bacteria (75\%), few Gram positive bacteria $(25 \%)$ and no fungal isolates among the study population. $K$. pneumoniae was the leading causal microbe in our study constituting $33.4 \%$ of all culture positive cases, which correlates with the National Neonatal Perinatal Data (NNPD) report of India [17] which reported $K$.pneumoniae as the most frequent pathogen (32.5\%).

All the isolated microbes in our study were sensitive to first line antibiotics except Elizabeth kingia meningoseptica and one episode of $K$. pneumoniae which were multi-drug resistant. Despite many published recommendations on the rational antibiotic therapy and multiple warnings regarding emerging antibiotic resistance, physicians tend to continue inappropriate and excessive use of antibiotics [18]. To avoid drug resistance one must practice the rational and judicious use of antibiotics. Initially one may choose a narrow spectrum drug when possible and a broad spectrum drug only in selected patients. One needs also to practice optimal duration of antibiotic therapy, stop antibiotics therapy within 2-3 days in patients whose blood or other fluid cultures are negative and continue longer only in patients with proven infection [19].

Among 23 VLBW infants with nosocomial sepsis, seven $(30.4 \%)$ died as compared to the 
mortality of four (5.8\%) out of 69 VLBW infants without nosocomial sepsis. Stoll BJ et al [13] and Karlowicz MG et al [20] reported that mortality risk was influenced by infecting pathogen. In our study, five among seven VLBW neonates with nosocomial sepsis who died grew Gram negative bacteria. This is in concurrence with the findings of Karlowicz et al [20] who reported a more fulminant illness and acute death in infants with Gram negative infections.

We found PICC line as an independent risk factor for Nosocomial sepsis in VLBW infants, but our study lacks sufficient power in view of small sample size. In contrast to the previous published data which shows a predominance of Gram positive infections in infants with Central venous catheters (CVC) we observed a predominance of Gram negative sepsis in this group of infants. The care of PICC lines can be improved by training and educating the physicians and nurses who insert and maintain catheters, using appropriate aseptic precautions at the time of PICC insertion and during usage, promptly removing the PICC line which is no longer essential or in case of any local site infection or if there is a suspicion of catheter related blood stream infection (CRBSI). Routine use of antiseptic/antibiotic impregnated central venous catheters and using antibiotic heparin lock in the CVC among VLBW infants to reduce the line-related infections also needs to be explored further.

We conclude that the risk of nosocomial sepsis among VLBW infants increases when the birth weight and gestational age decreases. Indwelling PICC line was identified to be the major risk factor for such infections. Gram negative bacteremia predominated in this group of infants with a significantly higher mortality as compared to Gram positive bacteremia. The significantly higher mortality of VLBW infants with nosocomial infections as compared to those without, stresses the need for strict and regular surveillance of this problem.

Declaration of conflicting interests: The authors declared no conflicts of interest with respect to the authorship and/or authorship of this article.

Funding: The authors received no financial support for the research and/or authorship of this article.

\section{REFERENCES}

1. Clark R, Powers R, White R, Bloom B, Sanchez P, Benjamin D. Nosocomial infection in the NICU: a medical complication or unavoidable problem? J Perinatol 2004; 24:382-8.

2. Elward AM, Hollenbeak CS, Warren DK, Fraser VJ. Attributable cost of nosocomial primary bloodstream infection in pediatric intensive care unit patients. Pediatrics 2005; 115:868-872.

3. Auriti C, Maccallini A, Di Liso G, Di Ciommo V, Ronchetti MP, Orzalesi M. Risk factors for nosocomial infections in a neonatal intensive-care unit. J Hosp Infect 2003; 53:25-30.

4. Brady MT. Health care-associated infections in the neonatal intensive care unit. Am J Infect Control 2005; 33:268-75.

5. Van der Zwet WC, Kaiser AM, et al. Nosocomial infections in a Dutch neonatal intensive care unit: surveillance study with definitions for infection specifically adapted for neonates. J Hosp Infect 2005; 61:300-11.

6. Lopez Sastre JB, CotoCotallo D, Fernandez Colomer B. Neonatal sepsis of nosocomial origin: an epidemiological study from the "Grupo de Hospitales Castrillo". J Perinat Med 2002; 30:14957.

7. Schelonka RL, Scruggs S, Nichols K, Dimmitt RA, carlo WA. Sustained reductions in neonatal nosocomial infection rates following a comprehensive infection control intervention. J Perinatol 2006; 26:176-179.

8. Bang AT, Bang RA, Baitule SB, Reddy HM, Deshmukh MD. Effect of home based neonatal care and management of sepsis on neonatal mortality: field trial in rural India. Lancet 1999; 354: 1955-61.

9. Schelonka RL, Infante AJ. Neonatal immunology. Semin Perinatol 1998; 22: 2-14.

10. Berger M. Complement deficiency and neutrophil dysfunction as risk factors for bacterial infections in newborns and the role of granulocyte transfusion in therapy. Rev Infect Dis 1990; 12 Suppl 4: S1401-S1409.

11. Gastmeier P, Geffers C, Schwab F, Fitzner J, Obladen M, Ruden $H$. Development of a surveillance system for nosocomial infections: the component for neonatal intensive care units in Germany. J Hosp Infect 2004; 57:126-31.

12. Brodie SB, Sands KE, Gray JE, et al. Occurrence of nosocomial bloodstream infections in six neonatal intensive care units. Pediatr Infect Dis $\mathrm{J}$ 2000; 19:56-65.

13. Fanaroff AA, Korones SB, Wright LL, et al. Incidence, presenting features, risk factors and 
significance of late onset septicemia in very low birth weight infants. The National Institute of Child Health and Human Development Neonatal Research Network. Pediatr Infect Dis J 1998; 17:593-598.

14. Stoll BJ, Gordon T, Korones SB, et al. Late-onset sepsis in very low birth weight neonates: a report from the National Institute of Child Health and Human Development Neonatal Research Network. J Pediatr 1996; 129:63-71.

15. Makhoul IR, Sujov P, Smolkin T, Lusky A, Reichman B. Epidemiological, Clinical, and Microbiological Characteristics of Late-Onset Sepsis Among Very Low Birth Weight Infants in Israel: A National Survey. Pediatrics 2002; 109:3439.

16. Stoll BJ, Hansen N, Fanaroff AA, et al. Late-Onset Sepsis in Very Low Birth Weight Neonates: The Experience of the NICHD Neonatal Research Network. Pediatrics 2002; 110:285-291.

17. National neonatal-Perinatal Database-2002-2003. AlIMS, New Delhi, for National Neonatology Forum, NNPD Network, India. Available at:www.newbornwhocc.org.

18. Goldmann DA, Weinstein RA, Wenzel RP, Tablan OC, Duma RJ, Gaynes RP. Strategies to prevent and control the emergence and spread of antimicrobial-resistant microorganisms in hospitals: a challenge to hospital leadership. JAMA 1996; 275:234-240.

19. Isaacs D. Rationing antibiotic use in neonatal units. Arch Dis Child 2000; 82: F1-F2.

20. Karlowicz MG, Buescher ES, Surka AE. Fulminant late-onset sepsis in a neonatal intensive care unit, 1988-1997, and the impact of avoiding empiric vancomycin therapy. Pediatrics. 2000; 106:13871390.

21. Sivanandan S, Amuchou S. Soraisham, Swarnam K. Choice and Duration of Antimicrobial Therapy for Neonatal Sepsis and Meningitis. Int $\mathrm{J}$ Ped $2011 ; 1-9$ 